\title{
Comparison of retinal vasodilator and constrictor responses in type 2 diabetes
}

\author{
Mary E. J. Lott, ${ }^{1}$ Julia E. Slocomb, ${ }^{2}$ Vikram Shivkumar, ${ }^{2}$ \\ Bruce Smith, ${ }^{1}$ Robert A. Gabbay, ${ }^{3}$ David Quillen, ${ }^{4}$ \\ Thomas W. Gardner ${ }^{5}$ and Kerstin Bettermann ${ }^{2}$ \\ ${ }^{1}$ Penn State Hershey Medical Center, Heart and Vascular Institute, Hershey, PA, \\ USA \\ ${ }^{2}$ Department of Neurology, Penn State Hershey Medical Center, Hershey, PA, USA \\ ${ }^{3}$ Penn State Institute for Diabetes and Obesity, Penn State College of Medicine, \\ Division of Endocrinology, Diabetes and Metabolism, Hershey, PA, USA \\ ${ }^{4}$ Department of Ophthalmology, Penn State Hershey Medical Center, Hershey, PA, \\ USA \\ ${ }^{5}$ Kellogg Eye Center, University of Michigan, Ann Arbor, MI, USA
}

\begin{abstract}
.
Purpose: The retinal blood vessels provide a unique way to directly examine the human microvasculature, which is frequently damaged in individuals with diabetes. Previous studies have demonstrated that retinal flickering light-induced vasodilation and hyperoxia-induced vasoconstriction may operate by enhancing or reducing similar vasoregulatory factor(s), but a comparison between these two provocative stimuli in individuals with diabetes has not been studied. The purpose of the study was to examine the association between retinal flickering light-induced vasodilation and retinal hyperoxia-induced vasoconstriction in type 2 diabetic subjects and in healthy controls.

Methods: Twenty men and women with type 2 diabetes and 10 men and women without diabetes between 21 and 75 years of age were recruited. Changes in retinal artery and vein diameters to flickering light and during hyperoxia (100\% oxygen) stimuli were measured on the same visit using a noninvasive retinal imaging device (Dynamic Vessel Analyzer, Imedos Inc., Germany).

Results: Compared with controls, diabetic subjects had impaired arterial vasodilator and vasoconstrictor responses to both flickering light and hyperoxia, respectively (both $\mathrm{p}<0.001)$. Merging both groups, an inverse correlation $(r=-0.56 ; p=0.003)$ between the retinal artery's responses to flickering light-induced vasodilation and hyperoxia-induced vasoconstriction was demonstrated independent of glucose or insulin levels. Conclusion: This suggests that both responses are attenuated to a similar degree in diabetic subjects and that the attenuation to both stimuli can be observed in retinal arteries and veins. This would suggest that similar vasoregulatory factor(s) might in part help to explain the retinal diameter responses between the two stimuli. One suggested common vasoregulator of vascular tone is nitric oxide; however, other factor(s) may be involved, which contribute to this association and require further research.
\end{abstract}

Key words: diabetes - flickering-induced vasodilation - hyperoxia - retinal reactivity - vasoconstriction - vasodilation

Acta Ophthalmol. 2012: 90: e434-e441

(c) 2012 The Authors

Acta Ophthalmologica $\odot 2012$ Acta Ophthalmologica Scandinavica Foundation

doi: $10.1111 / \mathrm{j} .1755-3768.2012 .02445 . x$

\section{Introduction}

Examination of the retinal blood vessels provides a direct view of the human microvasculature and allows the study of autoregulatory responses that are mediated via difference mechanisms (e.g. metabolic and myogenic) (Patton et al. 2006). Retinal structure and function is altered in individuals with diabetes (Pournaras et al. 2008) and is proposed to contribute to the pathogenesis of diabetic retinopathy (Kohner et al. 1995; Grunwald et al. 1996; Schmetterer \& Wolzt 1999). Thus, the study of retinal vascular function can provide important information about predisposition to vascular diseases associated with diabetes (Patton et al. 2006; Pournaras et al. 2008).

Vascular reactivity, which is the change in magnitude of a haemodynamic variable in response to a provocative stimulus, is a measure of vascular function. Flickering light and hyperoxia are two well-known provocative metabolic stimuli for the retinal vasculature (Pournaras et al. 2008). Flickering light stimulation involves a short series of light flashes for a specified time period (e.g. 15-64 seconds), which causes retinal blood vessels to vasodilate resulting in increased retinal blood flow and blood vessel 
diameter in healthy individuals (Formaz et al. 1997; Michelson et al. 2002; Dorner et al. 2003; Nagel et al. 2004). By contrast, retinal blood vessel diameter constricts and blood flow decreases in response to hyperoxia in healthy individuals (Riva et al. 1983; Kiss et al. 2002; Gilmore et al. 2005; Jean-Louis et al. 2005). Both vasodilator responses (Grunwald et al. 1984; Garhofer et al. 2004; Mandecka et al. 2007; Pemp et al. 2009) and vasoconstrictor responses are attenuated in diabetes (Gilmore et al. 2007; Justesen et al. 2010). However, whether a relationship exists between these two stimuli is unclear, as the majority of retinal studies have reported retinal vasoreactivity to either one or the other of these stimuli without direct comparison in vasoreactivity to both stimuli. An early study conducted in only three healthy subjects suggested that the vasoconstrictor response to hyperoxia compared with magnitude of the flickering light-induced vasodilation was greater (Formaz et al. 1997); This study had technical limitations in the set-up used for the administration of oxygen, which may have lead to changes in carbon dioxide levels, and used static instead of dynamic fundus photographs, which did not allow continuous measurements of changes in retinal vessel diameters.

Few studies have examined the potential underlying mechanisms that lead to retinal vasodilation and vasoconstriction by flickering light and hyperoxia. Animal and human retinal studies suggest that part of the flickering light-induced vasodilation can be explained by an increase in nitric oxide (Buerk et al. 1996; Kondo et al. 1997; Dorner et al. 2003). Peripheral and coronary blood flow studies in animals and humans suggest that part of the blood vessel vasoconstriction associated with hyperoxia can be explained through the increase of reactive oxygen species, which can reduce or quench nitric oxide availability (Rubanyi \& Vanhoutte 1986; Mak et al. 2002; McNulty et al. 2007). Therefore, both hyperoxia and flicker may evoke vasoconstriction and dilation in part via modulation (i.e. reduction or enhancement) of a similar vasoregulator factor (i.e. nitric oxide).

Thus, the purpose of the study was to examine the association between flickering light-induced vasodilation and hyperoxia-induced vasoconstriction in healthy and type 2 diabetic individuals. As both stimuli may modulate the vasoregulator factor, nitric oxide, we hypothesized that flickering light-induced vasodilation and hyperoxia-induced vasoconstriction may have similar magnitude changes and be inversely correlated in these populations. This information could be helpful in understanding the range of responsiveness in the human retinal vessels to common regulatory systems in health and disease.

\section{Methods}

\section{Subjects}

The study was approved by Penn State Hershey Medical Center's Institutional Review Board and followed the Tenets of the Declaration of Helsinki. Twenty men and women with type 2 diabetes and 10 men and women without diabetes between 21 and 75 years of age were enrolled into the study. Diabetic subjects were individuals diagnosed with diabetes by their physician. After signing informed consent, all subjects completed a medical history, physical exam and ocular screening, which included a measurement of visual acuity. Subjects had a normal eye examination with corrected acuity $20 / 30$ or better and intraocular pressures below $21 \mathrm{mmHg}$. Subjects were free from stroke, heart, lung and eye diseases (e.g. retinopathy, age-related macular degeneration or glaucoma) and from morbid obesity (body mass index (BMI) $>$ $40 \mathrm{~kg} / \mathrm{m}^{2}$ ) and were not pregnant. All subjects were nonsmokers, and controls had no history of hypertension.

\section{Experimental design}

The retinal diameter responses to flickering light and hyperoxia were measured during one visit with a 15 -min rest period between experimental trials (Fig. 1). The Dynamic Vessel Analyzer (DVA) measured retinal reactivity. Subjects refrained from alcohol, caffeine and exercise for $24 \mathrm{hr}$ prior to testing. Measurements were performed in a dimly lit room with temperature $68-70^{\circ}$ with subjects in a fasted state for approximately $10 \mathrm{hr}$. Individuals with type 2 diabetes refrained from using all medications in the morning of the study. Aspirin and nonsteroidal antiinflammatory medications were held for $72 \mathrm{hr}$ prior to the study. After a 15-min rest period, venous blood samples from the antecubital location were drawn for later analysis. The eye with the best visual acuity was dilated with one drop of tropicamide (1\%). If needed, phenylephrine $(2.5 \%)$ was additionally used to obtain optimal dilation.

\section{Experimental protocol}

An initial room air breathing period was used to allow stabilization of baseline parameters. Hyperoxia was induced by administrating $100 \%$ oxygen via a rubber mouthpiece, which was snugly fitted into the subject's mouth. Nares were clipped closed to ensure that the subject was breathing only through the mouth. The mouthpiece was connected to a six-inch tube which was then connected to a T-piece which had a one-way valve on either side for inhalation and exhalation. Oxygen was delivered through a reservoir bag, which connected to the one-way inhalation valve T-piece. While sitting, the subject's retinal

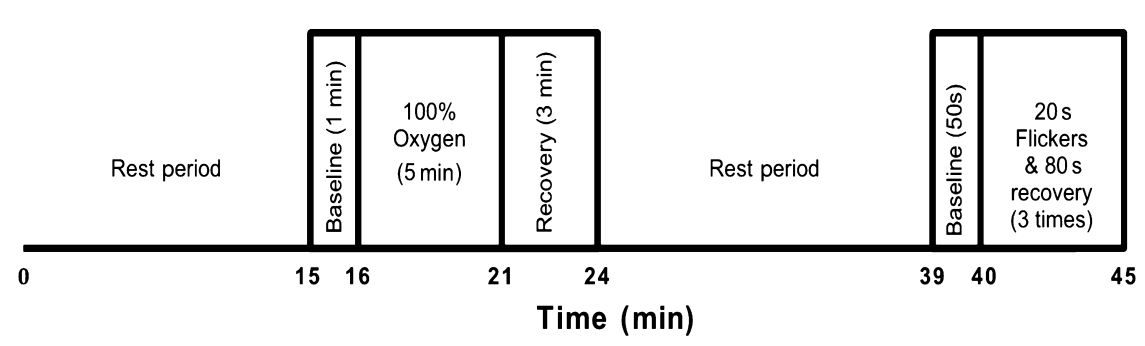

Fig. 1. Diagram of the experimental protocol. After a 15 -min rest period, retinal vascular diameters were measured at room air for $1 \mathrm{~min}$ followed by $100 \%$ oxygen for $5 \mathrm{~min}$. Retinal images were recorded during the 3-min recovery period back at room air. After a minimum rest period of $15 \mathrm{~min}$, retinal baseline diameters for the flicker trial were collected followed by three series of flicker (20 seconds) and recovery periods ( 80 seconds) for a total of 6 min. 
vessel diameters were imaged continuously during each stimulus using the DVA. Following $1 \mathrm{~min}$ of baseline with inhalation of room air, $100 \%$ oxygen was administered for $5 \mathrm{~min}$ followed by a 3-min recovery period back at room air. After a minimal rest period of $15 \mathrm{~min}$, flickering light measurements were conducted as the subject's retinal vessels were imaged continuously. After 50 seconds of baseline, the flickering light stimulus involved three cycles of light flashes (20 seconds) at a $12.5 \mathrm{~Hz}$ frequency followed by 80 seconds of a rest period between each series of light flashes (Mandecka et al. 2007; Nguyen et al. 2009). The entire measurement lasted 6 min. Blood pressure (BP) and heart rate (HR) were measured continuously during the studies. Additionally, end tidal $\mathrm{CO}_{2} \quad\left(\mathrm{ET}_{\mathrm{ET}} \mathrm{CO}_{2}\right)$ and finger oxygen saturation $\left(\mathrm{O}_{2 \mathrm{Sat}}\right)$ were continuously measured during the hyperoxia trial (See Fig. 1 for experimental protocol paradigm).

\section{Measurements}

Retinal vessel diameters

The DVA (Imedos Inc., Germany) uses a modified fundus camera (Zeiss FF450; Zeiss Jena, Germany) and a video recording unit. The system scans retinal diameters in real time, and vessel calibres can be analysed off-line (Garhofer et al. 2010). To obtain images, the subjects' fixation in the fundus camera was adjusted so that the optic nerve head was in the centre of the fundus monitor. The fundus camera focus and green background light were adjusted to provide sharp images on the fundus monitor. The region of interest was marked over a superior or inferior temporal retinal artery and vein one to two optic disc diameters from the optic nerve disc. This region of interest is measured 25 times per second, and measurements are reported in arbitrary units (AU). Eye-tracking technology in the DVA compensates for small eye movements. Throughout the testing, the subject was verbally urged to maintain his/her fixation on the fixation pointer within the DVA and to blink frequently to maintain crisp images. All images were stored on a VHS videotape recorder for off-line measurements of retinal artery and vein diameters. One observer analysed all measurements.
Haemodynamics (HR and BP)

Heart rate derived from the electrocardiogram and BP measured by use of a Finometer device (model 2300; Ohmeda, Boulder, CO, USA, confirmed by an automated sphygmomanometer, Dinamap, Critikon, Tampa, FL, USA) were measured continuously and collected on-line at $200 \mathrm{~Hz}$.

\section{Ventilatory parameters}

ET $\mathrm{CO}_{2}(\%)$ was measured continuously with a respiratory gas monitor during the hyperoxia trials (RGM 5250; Ohmeda, Madison, WI, USA). $\mathrm{O}_{2 \text { Sat }}(\%)$ was measured using a finger pulse oximeter (Model Biox 3740; Ohmeda, Louisville, CO, USA).

Plasma biomarkers

Fasting glucose, insulin, haemoglobin (HB) $\mathrm{A}_{1 \mathrm{C}}$ and lipid profile were measured using radioimmunoassays (Diagnostic Products Corp., Los Angeles, CA, USA).

\section{Statistical analysis}

For flickering light experimental trials, the resting baseline measurements were averaged from the last $15 \mathrm{sec}$ onds of resting conditions, and peak retinal dilation was captured from the last 3-10 seconds of the flickering stimuli and the first 3 seconds after the stimulus ended (Mandecka et al. 2007). The percentage change in vessel diameter was calculated comparing baseline retinal diameter with the diameter at a specific time point using the following equation: Per cent change in diameter $=\left(\left(\left(\right.\right.\right.$ diameter $_{\mathrm{x}}-$ diameter $\left._{\text {baseline }}\right) /$ diameter $\left._{\text {baseline }}\right) \times 100$ ) . $\mathrm{We}$ also measured maximal retinal vasoconstriction after peak diameter dilation (i.e. smallest diameter after peak dilation - averaged of $4 \mathrm{sec}$ onds). Lastly, we calculated the overall range of change in the retinal diameter known as relative amplitude (percent change in relative amplitude $=$ percent change in peak dilation $+\mid$ percent change in maximal vasoconstriction) (Nagel et al. 2004). For the hyperoxia trials, the last 30 seconds of resting baseline and last 15 seconds of $5 \mathrm{~min}$ of hyperoxia were measured and the percent change from baseline was calculated.

Two-tailed unpaired $t$-tests were used to compare demographic, anthropometric, blood samples and resting haemodynamic variables between the two groups (healthy controls and type 2 diabetic subjects). Analysis of variance models were fit to continuous outcomes to assess differences between the independent factors of group (controls and diabetic subjects) and retinal vessel (artery and vein), as well as the interaction of group and retinal vessel. With the groups' data merged, the correlations between two continuous variables (vascular reactivity responses to hyperoxia and flickering light stimuli) were assessed using Pearson's correlation coefficients and partial correlations adjusted for covariates (glucose and insulin). Results are presented as means \pm SD. A p $<0.05$ was regarded as significant.

\section{Results}

\section{Subject characteristics}

Groups were comparable in age, weight and BMI (Table 1). Most of the diabetic subjects were treated with oral diabetic medications $(87 \%)$ and several with combination therapy with long- and/or short-acting insulin $(20 \%)$. The majority of those with diabetes were also on anti-hypertension $(93 \%)$ and statin $(60 \%)$ therapy. Diabetic subjects had higher fasting glucose and $\mathrm{HbA}_{1 \mathrm{C}}$ levels compared with healthy controls (Table 1). Compared with control subjects, diabetic subjects tended to have a greater resting HR, but no differences in resting mean arterial pressure (MAP), systolic blood pressure (SBP) or diastolic blood pressure (DBP) were noted (Table 2). Resting retinal artery and vein diameters within groups were similar at baseline prior to the two stimuli (Controls: artery, $116 \pm 11$ $\mathrm{AU}$ and $116 \pm 6 \mathrm{AU}$; vein, $133 \pm 18$ $\mathrm{AU}$ and $136 \pm 20 \mathrm{AU}$, prehyperoxia and preflicker, respectively; Diabetic subjects: artery, $118 \pm 17 \mathrm{AU}$ and $118 \pm 15$ AU; Vein, $147 \pm 22$ and AU $148 \pm 24$ AU, prehyperoxia and preflicker, respectively, all $\mathrm{p}>0.05$ ).

\section{Hyperoxia-induced vasoconstriction}

Finger pulse $\mathrm{O}_{2 \text { Sat }}$ from baseline levels increased in both groups during hyperoxia (Controls: $98 \pm 1 \%$ to $100 \pm$ $1 \%, \quad \mathrm{p}=0.003$; Diabetic subjects: $98 \pm 0 \%$ to $99 \pm 0 \%, \mathrm{p}<0.001)$. Hyperoxia evoked similar artery and 
Table 1. Subject demographics, anthropometrics and fasting plasma blood biomarkers.

\begin{tabular}{|c|c|c|c|c|c|}
\hline & $\begin{array}{l}\text { Healthy } \\
\text { controls }\end{array}$ & $95 \% \mathrm{CI}$ & $\begin{array}{l}\text { Type } 2 \\
\text { Diabetes }\end{array}$ & $95 \% \mathrm{CI}$ & p-value \\
\hline Number of subjects & 10 & & 20 & & \\
\hline Number of women/men & $5 / 5$ & & $7 / 13$ & & \\
\hline Age (years) & $50 \pm 7$ & 45,55 & $56 \pm 9$ & 52,60 & 0.06 \\
\hline Weight (lbs.) & $205 \pm 41$ & 176,235 & $194 \pm 46$ & 169,212 & 0.50 \\
\hline $\operatorname{BMI}\left(\mathrm{kg} / \mathrm{m}^{2}\right)$ & $30.7 \pm 5.1$ & 27,34 & $30.4 \pm 5.5$ & 27,33 & 0.89 \\
\hline Fasting glucose $(\mathrm{mg} / \mathrm{dl})$ & $86 \pm 7$ & 80,91 & $115 \pm 30^{*}$ & 100,129 & 0.01 \\
\hline Fasting insulin (mg/dl) & $5 \pm 3$ & & $16 \pm 29$ & & 0.22 \\
\hline $\mathrm{HbA}_{1 \mathrm{C}}(\%)$ & $5.2 \pm 0.3$ & $5.0,5.4$ & $7.2 \pm 1.3^{*}$ & $6.6,7.8$ & 0.00 \\
\hline Duration diabetic (years) & & & $7.2 \pm 1.2$ & & \\
\hline Total Cholesterol (mg/dL) & $198 \pm 33$ & 175,222 & $176 \pm 35$ & 159,192 & 0.10 \\
\hline Low-Density Lipoprotein (mg/dl) & $131 \pm 29$ & 110,152 & $100 \pm 32 *$ & 87,114 & 0.01 \\
\hline High-Density Lipoprotein $(\mathrm{mg} / \mathrm{dl})$ & $47 \pm 12$ & 38,56 & $49 \pm 12$ & 43,55 & 0.70 \\
\hline Lipid Ratio & $4.2 \pm 1.5$ & $3.0,5.3$ & $3.8 \pm 1.0$ & $3.4,4.2$ & 0.30 \\
\hline Triglycerides (mg/dl) & $92 \pm 31$ & 71,114 & $130 \pm 60$ & 102,158 & 0.07 \\
\hline
\end{tabular}

$\mathrm{BMI}$, body mass index; $\mathrm{HbA}_{1 \mathrm{C}}$, haemoglobin $\mathrm{AlC}_{\mathrm{C}}$; $\mathrm{CI}$, confidence intervals; means $\pm \mathrm{SD}$.

* Significant difference between groups.

Table 2. Resting haemodynamics.

\begin{tabular}{lcrcrl}
\hline Subjects & Healthy controls & $95 \% \mathrm{CI}$ & Type 2 Diabetes & $95 \% \mathrm{CI}$ & p-value \\
\hline HR $(\mathrm{bpm})$ & $60 \pm 5$ & 56,63 & $68 \pm 10^{*}$ & 63,73 & 0.02 \\
MAP $(\mathrm{mmHg})$ & $93 \pm 8$ & 88,98 & $96 \pm 15$ & 92,100 & 0.36 \\
SBP $(\mathrm{mmHg})$ & $120 \pm 9$ & 114,126 & $129 \pm 15$ & 122,136 & 0.10 \\
DBP $(\mathrm{mmHg})$ & $80 \pm 7$ & 75,85 & $80 \pm 7$ & 77,83 & 0.99 \\
\hline
\end{tabular}

HR, heart rate; MAP, mean arterial pressure; SBP, systolic blood pressure; DBP, diastolic blood pressure; means $\pm \mathrm{SD}$; CI, confidence intervals.

* Significant difference between groups.

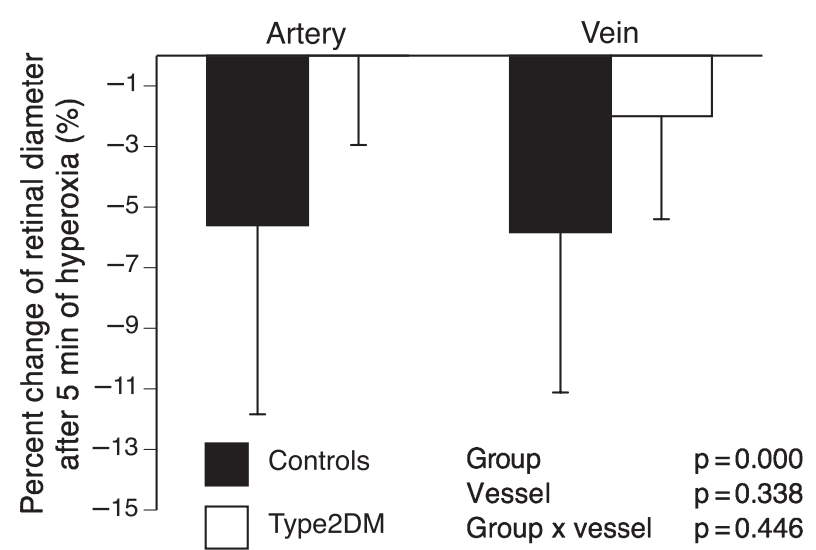

Fig. 2. Type 2 diabetes reduces retinal vascular vasoconstrictor responses to hyperoxia. Type 2 diabetic (Type2DM) subjects had attenuated retinal artery and vein vasoconstrictor responses to hyperoxia compared with controls (group effect, $\mathrm{p}<0.001$ ). vein vasoconstriction in the majority $(90 \%)$ of healthy controls, whereas artery and vein diameters decreased in only $50 \%$ of the diabetic subjects in response to oxygen. Compared with healthy subjects, diabetic subjects had significantly attenuated artery and levels in either group (controls: $5.3 \pm 0.5 \%$ to $5.4 \pm 0.5 \%, \quad \mathrm{p}=$ 0.10 ; diabetic subjects: $4.9 \pm 0.8 \%$ to $4.7 \pm 1.1 \% ; \mathrm{p}=0.34)$.

\section{Flickering Light-Induced vasodilation}

Flickering light evoked vasodilation in the majority in each group $(95 \%$ in controls and $91 \%$ in diabetic subjects), although the artery and vein peak vasodilator responses were significantly attenuated in the diabetic group compared with the healthy control group (Fig. 3A, group effect, $p<0.001)$. Retinal vein diameters increased to a greater extent at peak vasodilation compared with retinal artery diameters (vessel effect, $\mathrm{p}=0.022$ ).

Maximal vasoconstriction after peak vasodilation was similar in the two groups and between vessels (Fig. 3B). Compared with healthy controls, diabetic subjects had attenuations in their overall per cent change in relative amplitude for the artery and vein responses to flickering light (Fig. 3C, p $<0.001$ ). Flickering light stimuli did not significantly change resting HR, MAP, SBP or DBP in either group (Data not shown).

\section{Hyperoxia compared with flickering light reactivity responses}

The healthy control group had similar changes in magnitude ( $\%$ change) in the retinal artery and vein between the two different stimuli (artery, p = 0.87; and vein, $p=0.57$ ). Diabetics had a greater magnitude change to peak flicker than hyperoxia in the artery $(\mathrm{p}=0.03)$ but not the vein $(p=0.17)$, whereas their artery and vein responses to hyperoxia were smaller in magnitude to their relative amplitude responses to flicker in both vessels $(p<0.001$ and $p=0.005$, respectively). When controls and diabetic groups were combined, we observed a significant inverse relationship in the retinal arteries between the magnitude of the constrictor responses to hyperoxia and peak dilator responses to flickering light $(r=-0.56$, $\mathrm{p}=0.003$; Fig. 4A). We observed a similar relationship between magnitude of the constrictor responses to hyperoxia and overall change in relative amplitude to flickering light $(r=-0.60, \quad \mathrm{p}<0.001 ; \quad$ Fig. 4B $)$. vein constrictor responses to hyperoxia (Fig. 2, Group effect, $\mathrm{p}=0.000$ ).

Hyperoxia did not significantly change HR, MAP, SBP or DBP in either group (data not shown). $\mathrm{ETCO}_{2}$ did not significantly change after $5 \mathrm{~min}$ of hyperoxia from baseline 

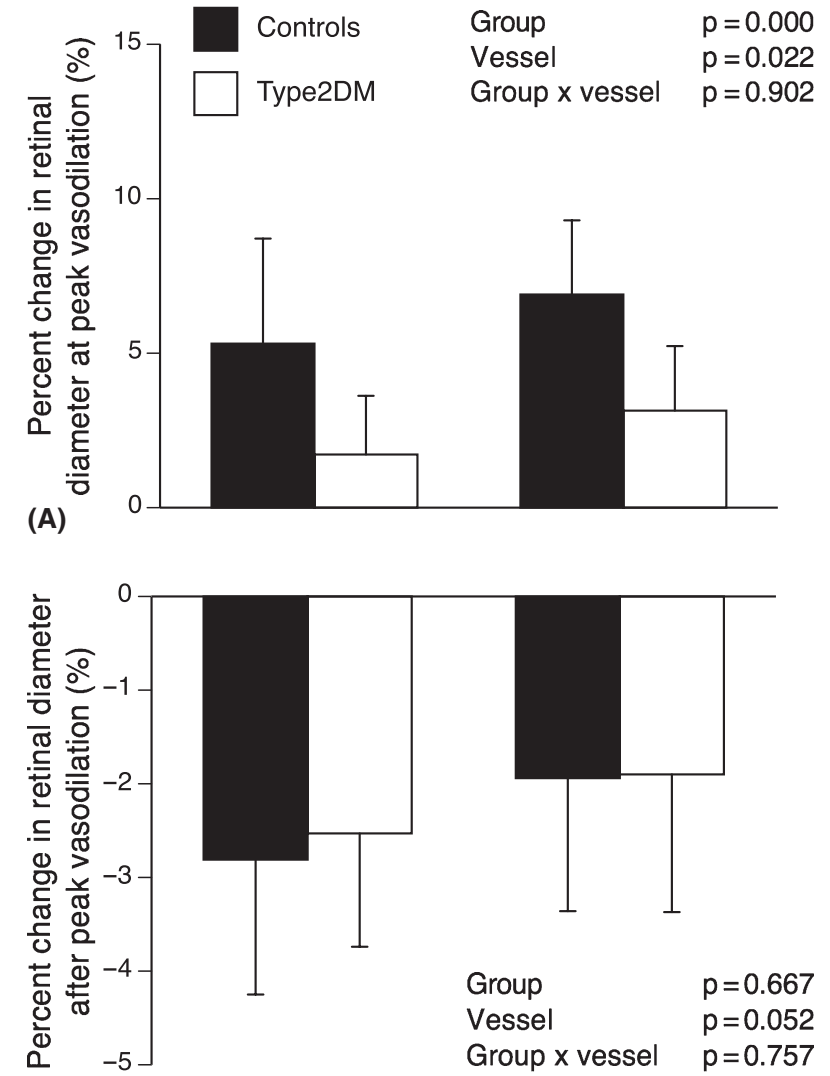

(B)

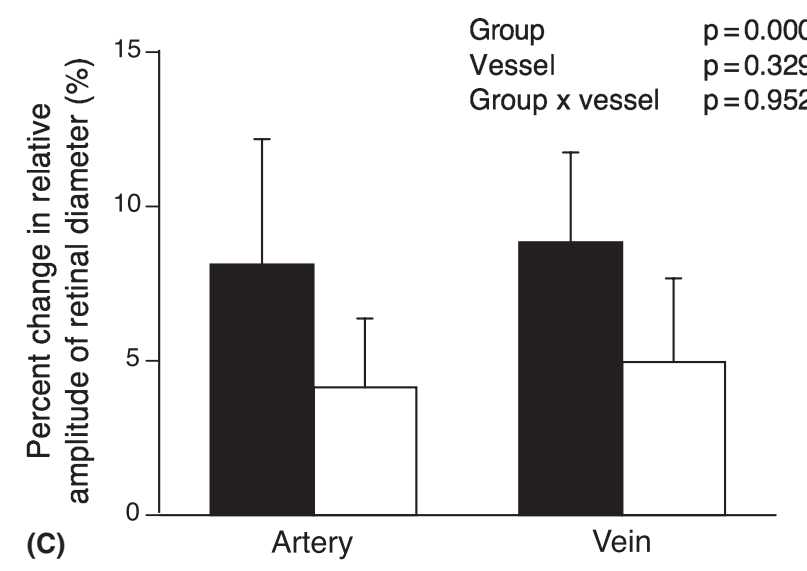

Fig. 3. A, B and C. Type 2 diabetes impairs retinal arterial dilation to flicker stimuli. Type 2 diabetic (Type2DM) subjects had attenuated retinal artery and vein vasodilation responses to flickering light-induced stimuli compared with controls (group effect, $\mathrm{p}<0.001$; Fig. 2A). Retinal vein vessels dilated to a greater degree than retinal artery vessels (vessel effect, $p=0.022$ ). There was no significant difference in maximal vasoconstriction after peak vasodilation in either vessel between groups or vessels (Fig. 2B); however, the percent change in relative amplitude in dilation showed attenuated responses in diabetic subjects compared with controls (group effect, p $<0.001 ;$ Fig. 2 C). $(\%=$ percent $)$.

These correlations were maintained when glucose and insulin levels were controlled for as covariates. A trend for a relationship between constrictor and dilator responses was observed for the retinal veins (peak dilator: $r=-0.35, \quad \mathrm{p}=0.06 ;$ and relative amplitude: $\quad r=-0.32, \quad \mathrm{p}=0.08$ )
(Fig. 4C,D); however, these vein correlations were not significant when glucose and insulin levels were controlled for as covariates. Lastly, we found no relationship between hyperoxia-induced vasoconstriction and the maximal vasoconstriction after peak flicker vasodilation.

\section{Discussion}

In this study, we examined (i) retinal dilation responses to flickering light, (ii) vasoconstrictor responses to hyperoxia, (iii) the magnitude of change to the stimuli, and (iv) the relationship between retinal dilator and constrictor responses to two stimuli in a group of healthy controls and type 2 diabetic subjects. We found attenuated vasodilator and vasoconstrictor responses in diabetic subjects to flickering light and hyperoxia and an inverse correlation between flickering light-induced vasodilation and hyperoxia-induced vasoconstriction. We propose that these data suggest that (i) dilation during flicker and constriction during hyperoxia may share common vasoregulatory factor(s) that are enhanced or reduced and that (ii) these common vasoregulatory factor(s) are abnormal in diabetes. As abnormal nitric oxide metabolism has been suggested to play a role in the progression of diabetic retinopathy (Maejima et al. 2001; Ozden et al. 2003; Izumi et al. 2006; Toda \& Nakanishi-Toda 2007; Ghosh et al. 2011), we speculate that nitric oxide may be the common vasoregulator factor, which is known to be altered in diabetic subjects, and mediates both stimulus pathways.

Our hyperoxia data confirmed results from previous studies in which diabetic subjects were noted to have attenuated artery and vein vasoconstrictor responses (Gilmore et al. 2007; Justesen et al. 2010). Previous studies reported greater reductions $(-10 \%$ to $-14 \%)$ in retinal artery and vein responses than those we noted $(5.6-5.8 \%)$ in our healthy older individuals. Prior studies were generally performed in younger subjects (Riva et al. 1983; Formaz et al. 1997; Kiss et al. 2002; Jean-Louis et al. 2005), and the different oxygen administration techniques used may have led to decreases in $\mathrm{CO}_{2}$, which may have enhanced the vasoconstrictor response in some of the earlier studies. However, the magnitude of change in artery vasoreactivity following hyperoxia in our study's control group was similar to the magnitude of change $(-8 \%)$ previously reported in middle-aged healthy subjects (Justesen et al. 2010). We also observed a similar magnitude in retinal artery and vein responses to hy- 
(A)
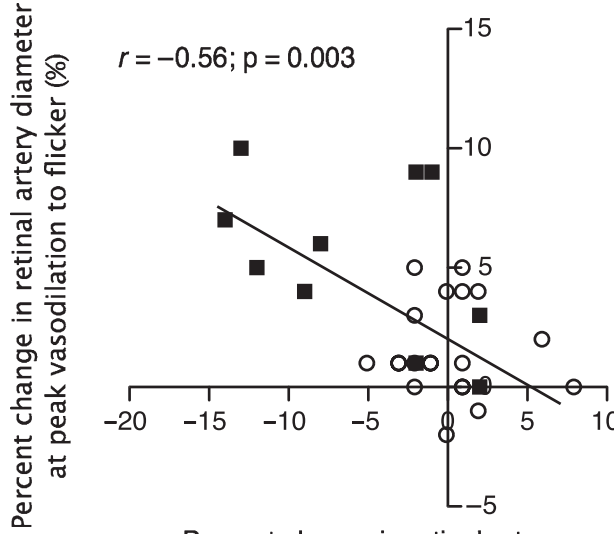

Percent change in retinal artery diameter to hyperoxia (\%)

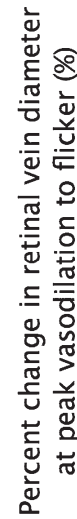

(C)

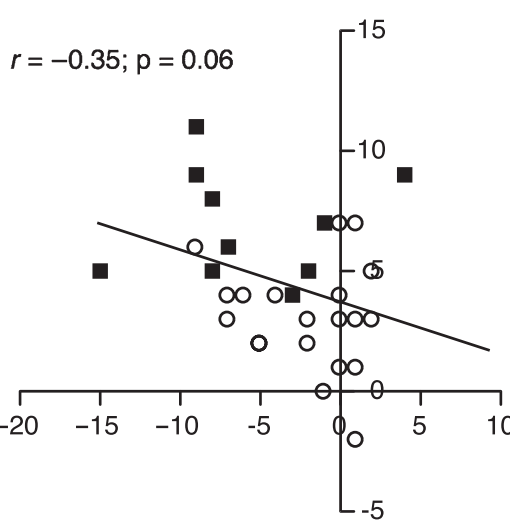

Percent change in retinal vein diameter to hyperoxia (\%)
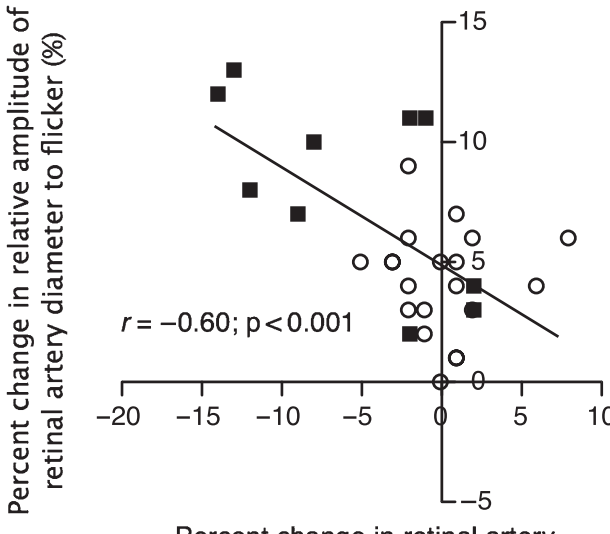

Percent change in retinal artery diameter to hyperoxia (\%)

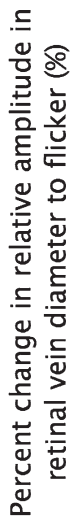

(D)

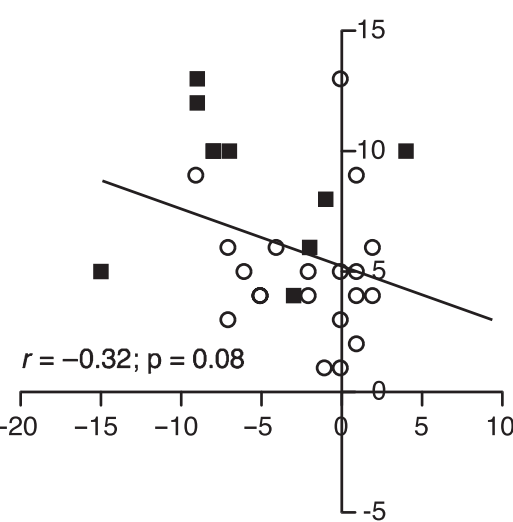

Percent change in retinal vein diameter to hyperoxia (\%)

Fig. 4. A, B, C and D. Correlation analysis between responses to hyperoxia and flicker stimulation. (A). Retinal artery diameter responses to hyperoxia vasoconstriction (horizontal line) and flickering light-induced vasodilation (vertical line) were significantly correlated by Pearson's method $(r=-0.56, \mathrm{p}=0.003)$. (B). Similar diameter associations were observed between hyperoxia vasoconstriction (horizontal line) and flicker light change in relative amplitude (vertical line) $(r=-0.60, \mathrm{p}<0.001)$. (C). Retinal vein diameter responses to hyperoxia vasoconstriction (horizontal line) and flickering-induced vasodilation (vertical line) were not significantly correlated by Pearson's method $(r=-0.35$, p $=0.06$ ). (D). Similar diameter associations were observed between hyperoxia vasoconstriction (horizontal line) and flicker light change in relative amplitude (vertical line) $(r=-0.32, \mathrm{p}=0.08)$. (White circles $=$ Type 2 diabetic subjects; Black squares $=$ control subjects $)$.

peroxia in the groups. Although the specific mechanisms for this are unclear, retinal arterial narrowing and changes in arterial compliance may contribute to small vessel stiffness (Cheung et al. 2007) and thus lead to attenuated arterial responses to hyperoxia in diabetic individuals.

Our flicker data confirmed previous studies examining retinal vessel responses to flicker in which normal subjects demonstrate robust diameter and blood flow responses (Formaz et al. 1997; Michelson et al. 2002; Dorner et al. 2003; Nagel et al. 2004). In contrast, disease states such as diabetes demonstrate attenuated dilator responses (Garhofer et al. 2004; Mandecka et al. 2007; Nguyen et al. 2009; Pemp et al. 2009). As noted earlier, the attenuated flicker response in diabetic retinal vessels has been suggested to be partly due to reduced nitric oxide (Schmetterer et al. 1997).

Recent retinal autoregulation studies (Bek et al. 2008; Skov Jensen et al. 2011) have demonstrated an interaction between two stimuli (flickering light and isometric handgrip) in healthy and diabetic subjects, suggesting the importance of examining responses to several stimuli to further understand mechanisms of retinal vascular dysfunction. Thus, our study examined whether a relationship exists between hyperoxia and flickering light in further understanding vascular function in healthy and diabetic subjects. We observed similar changes in magnitude of the retinal artery and vein responses in both the control and diabetic groups. In our study, the magnitude of the hyperoxia-induced vasoconstriction $(-5.6 \%$ to $-5.8 \%)$ was similar to the magnitude in vasodilation (5.3-6.9\%) following light flicker stimulation of the arteries and veins in the healthy control group. This finding is in contrast to a previous study of healthy young subjects $(n=3)$, which demonstrated a greater magnitude change in hyperoxia compared to flicker (Formaz et al. 1997). Reasons for these differences may be due to technical differences (administration set-up for hyperoxia, use of fundus photographs, and duration of flickering light stimulus) and differences in the group's ages. In our 
study, diabetic's retinal diameter responses were also similarly attenuated in their magnitude response to hyperoxia and flicker. These autoregulatory vasodilator and vasoconstrictor impairments in individuals with diabetes may increase their predisposition to microvascular complications such as diabetic retinopathy (Kohner et al. 1995).

In our study, a common vasoregulatory factor(s) may help to explain the $30 \%$ of the variance between the two stimuli in the retinal arterial responses independent of glucose and insulin levels. We speculated that this common factor(s) includes nitric oxide. Animal and human retinal studies suggest that part of the flickering light-induced vasodilation can be explained by an increase in the production of nitric oxide (Buerk et al. 1996; Kondo et al. 1997; Dorner et al. 2003), whereas animal and human peripheral and coronary studies suggest that part of the blood vessel vasoconstriction associated with hyperoxia can be explained through the increase in reactive oxygen species, which can reduce nitric oxide availability (Rubanyi \& Vanhoutte 1986; Mak et al. 2002; McNulty et al. 2007). Yet, it must be noted that hyperoxia and flicker may involve different pathways and multiple intracellular mechanisms that may be altered in diabetes (Kim J 2006). Animal and human studies suggest that besides nitric oxide, superoxide generation (Rubanyi \& Vanhoutte 1986; Zhilyaev et al. 2003; McNulty et al. 2007), endothelin-1 (Takagi et al. 1996; Higgins et al. 1998; Zhu et al. 1998; Dallinger et al. 2000; Kawamura et al. 2002), thromboxane A (Takagi et al. 1996; Zhu et al. 1998), 20HETE (Takagi et al. 1996; Zhu et al. 1998), cytochrome-P450 (Takagi et al. 1996; Zhu et al. 1998) and prostanoids (Yu et al. 2001) may be contributors to hyperoxia constrictor responses. Although animal and human studies suggest that part of the flickering light-induced vasodilation responses can be explained by an increase in the production of nitric oxide (Buerk et al. 1996; Kondo et al. 1997; Dorner et al. 2003), other mechanisms such as an increase in potassium may also play a role in the response to flicker stimulus (Buerk et al. 1995).

In our study, the majority of the diabetic subjects were using statin therapy. Human and animal studies suggest the use of statins may be associated with improvements in macrovascular (Bellia A 2010) and microvascular (Nagaoka et al. 2007) function possibly through an antioxidant or anti-inflammatory mechanism. In our study, despite the use of statins in the diabetic subjects, their retinal reactivity to hyperoxia and flickering light was still greatly impaired compared with that in healthy controls. Further studies are needed to fully explore the effect of statins on retinal reactivity in diabetes.

Viewing the fundus of the eye allows direct simultaneous measurements of arterial and venous responses. Early studies showed that both retinal veins and arteries could respond to vasodilator and vasoconstrictor stimuli. Studies have typically reported responses in both vessels but have not compared the magnitude of change between vessels. In our study, the magnitude of change in retinal artery and vein diameter responses were similar during hyperoxia. During flickering light stimulation, the magnitude of peak dilation was greater in the retinal veins compared with that in the retinal arteries, whereas the magnitude of maximal vasoconstriction after peak vasodilation trended to be greater in the retinal arteries compared with that in the retinal veins. It is difficult to compare the magnitude of these responses with prior studies using these stimuli owing to differences in duration of the stimuli given and frequency of the light stimulus used. In our study, the retinal vessels chosen were within 1-2 discs diameters from the optic disc. Typically, compared with retinal veins, retinal arteries in this area have a thicker muscle layer and may exhibit a greater vasoconstrictor response effect to pressure stimuli (Anad-Apte \& Hollyfield 2011). As endothelial cells line both retinal arteries and veins (Anad-Apte \& Hollyfield 2011), greater vein vasodilator effects are unclear compared with artery. Thus, comparison of retinal arteries and veins to various stimuli and metabolites merits further investigation.

Limitations: First, we lacked measurement of arterial blood gases during hyperoxia. Second, the slight nonsignificant reduction in ${ }_{\mathrm{ET}} \mathrm{CO}_{2}$ with hyperoxia could have contributed to the vasoconstrictor effect seen; however, this was only seen in our diabetic subjects who had an attenuated vasoconstrictor response. Third, to obtain sufficiently dilated pupils, we had to use phenylephrine in some of our subjects, but the findings were consistent when these individuals were excluded from the analysis. Thus, we do not feel that this affected our results. Fourth, the order of our trials had the potential of influencing our results; however, we feel that this is unlikely due to short duration of hyperoxia (5 $\mathrm{min})$, a standard minimum of time between trials (15 min), and that there was no significant difference between resting retinal diameters prior to any stimuli. Lastly, we were only able to measure diameters, not blood flow, so it is possible that velocity may be altered differently by the two stimuli; however, we could not measure retinal blood velocity.

In summary, the attenuated magnitudes of flicker light-induced vasodilation and hyperoxia-induced vasoconstriction on the same visit in individuals with type 2 diabetes are comparable. Similar common vasoregulatory factor(s) may help to explain over $30 \%$ of the variance between the two stimuli in the retinal arteries. One suggested common vasoregulator factor is nitric oxide; however, other factor(s) may be involved which contribute to this association. Further studies are needed to determine which factors alter endothelial function in diabetes which may allow the development of new interventions to prevent early stages of atherosclerosis and retinopathy associated with diabetes. Finally, the measured vascular responses may reflect direct changes in physiology of blood vessels and/or the retinal neuroglial structures.

\section{Acknowledgements}

Preliminary results of the study were presented as an abstract: "Lott, MEJ, Bettermann, K, Slocomb, JE, Smith, J, Gabbay, R, Gardner, T. Retinal Reactivity in Type 2 Diabetes. Experimental Biology, Anaheim, CA, 24-28 April 2010. We would like to thank Surju Patel, Cheryl Blaha and Jessica Mast for their help with the study. We would like to thank the subjects for participating in the study as well as the nursing staff of the Penn State Hershey Medical Center's Clinical Research Center for technical assistance. We would also like to especially thank the Pennsylvania Lions Sight Conservation and Eye Research Foundation for their generous contribution in obtaining the DVA instrument. Support 
was provided by a grant from the Pennsylvania Tobacco Settlement Funds. The authors declare no conflict of interest.

\section{References}

Anand-Apte B \& Hollyfield JG (2011): Development anatomy of the retinal and choroidal vasculature. In: Besharse JC \& Bok D (eds.). The retina and its disorders. San Diego, CA, USA: Academic Press (Elsevier Ltd): 179-194. Bek T, Hajari J \& Jeppesen P (2008): Interaction between flicker-induced vasodilatation and pressure autoregulation in early retinopathy of type 2 diabetes. Graefes Arch Clin Exp Ophthalmol 246: 763-769.

Bellia A, Rizza S, Galli A, Fabiano R, Donadel G, Lombardo MF, Cardillo C, Sbraccia P, Tesauro M \& Lauro D (2010): Early vascular and metabolic effects of rosuvastatin compared with simvastatin in patients with type 2 diabetes. Atherosclerosis 210: 199-201.

Buerk DG, Riva CE \& Cranstoun SD (1995): Frequency and luminance-dependent blood flow and $\mathrm{K}+$ ion changes during flicker stimuli in cat optic nerve head. Invest Ophthalmol Vis Sci 36: 2216-2227.

Buerk DG, Riva CE \& Cranstoun SD (1996): Nitric oxide has a vasodilatory role in cat optic nerve head during flicker stimuli. Microvasc Res 52: 13-26.

Cheung N, Islam FM, Jacobs DR Jr et al. (2007): Arterial compliance and retinal vascular caliber in cerebrovascular disease. Ann Neurol 62: 618-624.

Dallinger S, Dorner GT, Wenzel R, Graselli U, Findl O, Eichler HG, Wolzt M \& Schmetterer L (2000): Endothelin-1 contributes to hyperoxia-induced vasoconstriction in the human retina. Invest Ophthalmol Vis Sci 41: 864869.

Dorner GT, Garhofer G, Kiss B, Polska E, Polak K, Riva CE \& Schmetterer L (2003): Nitric oxide regulates retinal vascular tone in humans. Am J Physiol Heart Circ Physiol 285: H631-H636.

Formaz F, Riva CE \& Geiser M (1997): Diffuse luminance flicker increases retinal vessel diameter in humans. Curr Eye Res 16: 1252-1257.

Garhofer G, Zawinka C, Resch H, Kothy P, Schmetterer L \& Dorner GT (2004): Reduced response of retinal vessel diameters to flicker stimulation in patients with diabetes. $\mathrm{Br} \mathbf{J}$ Ophthalmol 88: 887-891.

Garhofer G, Bek T, Boehm AG et al. (2010): Use of the retinal vessel analyzer in ocular blood flow research. Acta Ophthalmol 88: 717-722.

Ghosh A, Sherpa ML, Bhutia Y, Pal R \& Dahal S (2011): Serum nitric oxide status in patients with type 2 diabetes mellitus in Sikkim. Int $\mathbf{J}$ App Basic Med Res 1: 31-35.

Gilmore ED, Hudson C, Preiss D \& Fisher J (2005): Retinal arteriolar diameter, blood velocity, and blood flow response to an isocapnic hyperoxic provocation. Am J Physiol Heart Circ Physiol 288: H2912-H2917.

Gilmore ED, Hudson C, Nrusimhadevara RK, Harvey PT, Mandelcorn M, Lam WC \& Devenyi RG (2007): Retinal arteriolar diameter, blood velocity, and blood flow response to an isocapnic hyperoxic provocation in early sight-threatening diabetic retinopathy. Invest Ophthalmol Vis Sci 48: 1744-1750.

Grunwald JE, Riva CE, Petrig BL, Sinclair SH \& Brucker AJ (1984): Effect of pure O2breathing on retinal blood flow in normals and in patients with background diabetic retinopathy. Curr Eye Res 3: 239-241.

Grunwald JE, DuPont J \& Riva CE (1996): Retinal haemodynamics in patients with early diabetes mellitus. Br J Ophthalmol 80: 327331 .
Higgins RD, Hendricks-Munoz KD, Caines VV, Gerrets RP \& Rifkin DB (1998): Hyperoxia stimulates endothelin-1 secretion from endothelial cells; modulation by captopril and nifedipine. Curr Eye Res 17: 487-493.

Izumi N, Nagaoka T, Mori F, Sato E, Takahashi A \& Yoshida A (2006): Relation between plasma nitric oxide levels and diabetic retinopathy. Jpn J Ophthalmol 50: 465-468.

Jean-Louis S, Lovasik JV \& Kergoat H (2005): Systemic hyperoxia and retinal vasomotor responses. Invest Ophthalmol Vis Sci 46: 1714-1720

Justesen BL, Mistry P, Chaturvedi N, Thom SA, Witt N, Kohler D, Hughes AD \& Sjolie AK (2010): Retinal arterioles have impaired reactivity to hyperoxia in type 1 diabetes. Acta Ophthalmol 88: 453-457.

Kawamura H, Oku H, Li Q, Sakagami K \& Puro DG (2002): Endothelin-induced changes in the physiology of retinal pericytes. Invest Ophthalmol Vis Sci 43: 882-888.

Kim JA, Montagnani M, Koh KK \& Quon MJ (2006): Reciprocal relationships between insulin resistance and endothelial dysfunction: molecular and pathophysiological mechanisms. Circulation 113: 1888-1904.

Kiss B, Polska E, Dorner G et al. (2002): Retinal blood flow during hyperoxia in humans revisited: concerted results using different measurement techniques. Microvasc Res 64: 75-85.

Kohner EM, Patel V \& Rassam SM (1995): Role of blood flow and impaired autoregulation in the pathogenesis of diabetic retinopathy. Diabetes 44: 603-607.

Kondo M, Wang L \& Bill A (1997): The role of nitric oxide in hyperaemic response to flicker in the retina and optic nerve in cats. Acta Ophthalmol Scand 75: 232-235.

Maejima K, Nakano S, Himeno M et al. (2001): Increased basal levels of plasma nitric oxide in Type 2 diabetic subjects. Relationship to microvascular complications. J Diabetes Complications 15: 135-143.

Mak S, Egri Z, Tanna G, Colman R \& Newton GE (2002): Vitamin C prevents hyperoxiamediated vasoconstriction and impairment of endothelium-dependent vasodilation. Am J Physiol Heart Circ Physiol 282: H2414H2421.

Mandecka A, Dawczynski J, Blum $\mathrm{M}$ et al. (2007): Influence of flickering light on the retinal vessels in diabetic patients. Diabetes Care 30: $3048-3052$.

McNulty PH, Robertson BJ, Tulli MA, Hess J, Harach LA, Scott S \& Sinoway LI (2007): Effect of hyperoxia and vitamin $\mathrm{C}$ on coronary blood flow in patients with ischemic heart disease. J Appl Physiol 102: 2040-2045.

Michelson G, Patzelt A \& Harazny J (2002): Flickering light increases retinal blood flow. Retina 22: 336-343.

Nagaoka T, Hein TW, Yoshida A \& Kuo L (2007): Simvastatin elicits dilation of isolated porcine retinal arterioles: role of nitric oxide and mevalonate-rho kinase pathways. Invest Ophthalmol Vis Sci 48: 825-832.

Nagel E, Vilser W \& Lanzl I (2004): Age, blood pressure, and vessel diameter as factors influencing the arterial retinal flicker response. Invest Ophthalmol Vis Sci 45: 1486-1492.

Nguyen TT, Kawasaki R, Wang JJ, Kreis AJ, Shaw J, Vilser W \& Wong TY (2009): Flicker light-induced retinal vasodilation in diabetes and diabetic retinopathy. Diabetes Care 32: 2075-2080

Ozden S, Tatlipinar S, Bicer N, Yaylali V, Yildirim C, Ozbay D \& Guner G (2003): Basal serum nitric oxide levels in patients with type 2 diabetes mellitus and different stages of retinopathy. Can J Ophthalmol 38: 393-396.
Patton N, Aslam TM, MacGillivray T, Deary IJ, Dhillon B, Eikelboom RH, Yogesan K \& Constable IJ (2006): Retinal image analysis: concepts, applications and potential. Prog Retin Eye Res 25: 99-127.

Pemp B, Garhofer G, Weigert G, Karl K, Resch H, Wolzt M \& Schmetterer L (2009): Reduced retinal vessel response to flicker stimulation but not to exogenous nitric oxide in type 1 diabetes. Invest Ophthalmol Vis Sci 50: 40294032

Pournaras CJ, Rungger-Brandle E, Riva CE, Hardarson SH \& Stefansson E (2008): Regulation of retinal blood flow in health and disease. Prog Retin Eye Res 27: 284-330.

Riva CE, Grunwald JE \& Sinclair SH (1983): Laser Doppler Velocimetry study of the effect of pure oxygen breathing on retinal blood flow. Invest Ophthalmol Vis Sci 24: 47-51.

Rubanyi GM \& Vanhoutte PM (1986): Superoxide anions and hyperoxia inactivate endothelium-derived relaxing factor. Am J Physiol Heart Circ Physiol 250: H822-H827.

Schmetterer L \& Wolzt M (1999): Ocular blood flow and associated functional deviations in diabetic retinopathy. Diabetologia 42: $387-$ 405 .

Schmetterer L, Findl $O$, Fasching $\mathrm{P}$ et al. (1997): Nitric oxide and ocular blood flow in patients with IDDM. Diabetes 46: 653-658.

Skov Jensen P, Jeppesen P \& Bek T (2011): Differential diameter responses in macular and peripheral retinal arterioles may contribute to the regional distribution of diabetic retinopathy lesions. Graefes Arch Clin Exp Ophthalmol 249: 407-412.

Takagi C, King GL, Takagi H, Lin YW, Clermont AC \& Bursell SE (1996): Endothelin-1 action via endothelin receptors is a primary mechanism modulating retinal circulatory response to hyperoxia. Invest Ophthalmol Vis Sci 37: 2099-2109.

Toda N \& Nakanishi-Toda M (2007): Nitric oxide: ocular blood flow, glaucoma, and diabetic retinopathy. Prog Retin Eye Res 26: 205-238.

Yu DY, Su EN, Cringle SJ, Schoch C, Percicot CP \& Lambrou GN (2001): Comparison of the vasoactive effects of the docosanoid unoprostone and selected prostanoids on isolated perfused retinal arterioles. Invest Ophthalmol Vis Sci 42: 1499-1504.

Zhilyaev SY, Moskvin AN, Platonova TF, Gutsaeva DR, Churilina IV \& Demchenko IT (2003): Hyperoxic vasoconstriction in the brain is mediated by inactivation of nitric oxide by superoxide anions. Neurosci Behav Physiol 33: 783-787.

Zhu Y, Park TS \& Gidday JM (1998): Mechanisms of hyperoxia-induced reductions in retinal blood flow in newborn pig. Exp Eye Res 67: 357-369.

Received on September 21st, 2011.

Accepted on March 13th, 2012.

Correspondence:

Mary Lott, PhD, MSN

Penn State Heart and Vascular Institute

The Milton S. Hershey Medical Center

Mail Code H047

500 University Drive

Hershey, PA 17033-2390, USA

Tel: + 17175311473

Fax: + 17175311792

Email: mlott@hmc.psu.edu 